\title{
PREDICTION OF THERMAL GROWTH IN A HIGH-SPEED SPINDLE BY CONSIDERING THERMO-MECHANICAL BEHAVIOR
}

\author{
E. Yuksel ${ }^{1}$, E. Budak ${ }^{1 *}$, E. Ozlu ${ }^{1}$, A. Oral ${ }^{2}$, F. Igrek², F. Tosun ${ }^{2}$ \\ ${ }^{1}$ Manufacturing Research Laboratory, Sabanci University, Tuzla, Istanbul 34956, Turkey \\ ${ }^{2}$ Igrek Makina Inc., OSB A.O. Sonmez Bulv. No:10 16140 Bursa, Turkey \\ *Corresponding author; e-mail: ebudak@sabanciuniv.edu
}

\begin{abstract}
Continuous rotation of spindle bearings and motor cause thermally induced structural deformations and thermal growth, which is one of the main reasons for machining errors. A positive feedback loop between bearing preload and heat generation causes preload variations in spindle bearings. These preload variations demonstrate a nonlinear transient behavior until the gradual expansion of outer bearing rings after which the thermally induced preload variation behaves steadily. In this study, a Finite Element (FE) framework is presented for predicting steady preload variation on spindle bearings. The method involves a thermal loading model and a transient contact analysis. In the contact analysis phase bearing contact deformations (penetration and sliding) and pressure are predicted by considering contact algorithms in an FE software. A transient spindle simulation in FE is employed to predict the bearing temperature and thermal spindle growth by using the proposed method. The performance of the method is demonstrated on a spindle prototype through bearing temperature and thermal deformation measurements. Results show that the proposed method can be a useful tool for spindle design and improvements due to its promising results and speed without the need for tests.
\end{abstract}

\section{Keywords:}

Spindle; Bearing Preload; Thermal; Contact

\section{INTRODUCTION}

Motorized spindles are critical elements in high-speed machining applications, and the required stiffness is maintained by preloaded bearings [1]. High-speed operations cause excessive heat production in spindle structures resulting in unsteady thermal behavior and preload variations [1]. The preload variations are due to the positive feedback between the preload and the heat loops [2], which results in a transient state on the thermal behavior of preloaded bearings. Contact surfaces on bearings function as fundamental elements to trigger the preload and heat feedback loop [3]. Bearing contact surfaces experience quasistatic and rolling contact conditions. The bearing contacts are classified as quasistatic since there is usually no abrupt change in contact conditions [4]. A quasistatic contact problem involves the contact displacements and contact forces as functions of time for a prescribed load history [5].

In general, this positive preload and heat feedback loop develops as the following. First, the rolling element temperature in the bearing rapidly increases due to friction and increases the bearing preload. At this early stage, inner bearing rings expand while outer bearing rings remain nearly unchanged since the outer ring surrounding act as heat sinks in a spindle assembly. Subsequently, the thermally induced preload causes more heat production in bearings, and the rolling elements expand further. This continued rolling element expansion finally leads to temperature rise on the outer rings, and eventually their gradual expansion creating a new steady-state behavior for the thermally induced bearing preload. The thermal behavior of bearing preload is explained at the assembly level so far.

In preloaded spindle bearings, the load history depends on the preload and heat feedback loop and demonstrates a nonlinear behavior. The rotating frame of spindle bearings results in two types of rolling contact conditions [6]: penetration and sliding which is associated with friction [4]. The contact penetration is predefined according to ISO standards [7] and given in the form of static load rating in bearing manufacturer catalogs. Permanent deformations may appear in rolling elements and raceways of rolling bearings under static loads. These static loads are adjusted according to the maximum allowable contact stress magnitudes. For instance, the maximum allowable contact stress for self-aligning ball bearings is $4600 \mathrm{MPa}$ while it is $4200 \mathrm{MPa}$ for all other ball bearings in ISO standards [7]. According to these contact stress limits, a total permanent deformation of $0.01 \%$ of the rolling element diameters may occur in spindle bearings. The contact penetration in 
spindle bearings is a function of bearing diameter (especially roller diameter) according to ISO-76:2006 [7] and can be predicted. This is important for two aspects. First, if one would like to model spindle bearing behavior by employing computer methods (FE, FDM, etc.), the contact algorithms will use Hertz- Signorini-Moreau (HSM) conditions which are based on the impenetrability of the contacted pairs [6]. However, the penalty-based methods for contact algorithms (the pure penalty and the augmented penalty method) allow penetration between the contacting bodies, and the penetration can be adjusted according to the rolling bearing diameters during the simulations. Otherwise, generally, $0.1 \%$ penetration is assigned automatically in most software [6] which may lead to misleading results in calculation of the contact penetration and sliding in simulations. Second, contact penetration causes a radial force on bearings. This radial force is a function of radial stiffness of bearings and contact penetration [8]. One must consider contact conductivity of rolling elements and spindle speed while computing contact sliding of spindle bearings. This is significant as the radial force acting on the bearings affects the contact conductance coefficient of ball bearings [8], and during the simulations, this boundary condition becomes the function of contact sliding. Thus, contact penetration becomes the function of contact sliding for spindle bearings, and one must consider this fact while calculating thermal deformations and related preload variations on spindle bearings.

Different solution methods (analytical and numerical) are employed to obtain spindle bearing thermal deformations by considering various aspects. For analytical approaches, some examples are as follows. Yan et al. [9] employed a hollow cylinder to predict raceway expansions by employing a Timoshenko beam and Hertz contact theory. Similarly, Zhang et al. [10] used elastic Hertz contact theory for bearing stress analyses and thermal deformation calculations. For the examples of the numerica approaches, Li et al. [11] used their bearing stiffness model in FEA for thermal expansion. Holkup et al. [12] employed an equivalent $\mathrm{FE}$ model for bearing rollers that simultaneously affects the thermal deformation of bearings. Even though, the contact penetration effect is limited, they consider bearing contact penetration while disregards the effect of contact sliding. None of these approaches considers the total contact displacement, i.e. penetration and sliding, while calculating the thermal bearing deformation of spindle bearings which is generally responsible for $15-45 \%$ of the total spindle deflection [2] However, contact sliding is directly associated with friction which is the most influential parameter on bearing preload variation [8] and dominantly effects on thermal deformation on spindle bearings compared to contact penetration.

In this study, a practical FE framework is proposed to predict bearing thermal deformation and preload variations by considering bearing contact penetration and sliding in spindle assemblies. The method involves direct application of spindle assembly boundary conditions onto bearings for thermal loading in a transient contact analysis to predict the steady-state phase preload variation by using contact algorithms in an FE software. Subsequently, the thermal FE analyses on a spindle structure using the proposed method are employed to obtain bearing temperature, thermal growth and finally the preload variations. In the therma transient spindle analyses, the steady bearing preload variation is used to reduce computational time by avoiding the preload heat loop iterations. This choice results in relatively higher transient bearing temperature and spindle thermal growth for operating periods shorter than 30 minutes. However, the proposed method does not require calibration tests or numerical iterations. Therefore, the proposed framework can be a useful and practical tool at the early design stage.

This article is organized as follows: the proposed method is explained in Section 2. The application of the proposed method on a spindle assembly is shared in Section 3. The comparison of the proposed method and experimental results are presented in Section 4. Lastly, the conclusions are listed in Section 5.

\section{THE BEARING CONTACT AND PRELOAD PREDICTION METHOD}

The proposed method includes a transient contact analysis stage for spindle bearing assemblies (bearings and spacers) since the thermal load is not distributed equally in different bearing configurations. In this analysis roller and raceway contact is represented by contact penetration and sliding. This contact analysis aims to detect the steady preload variation of bearings after the gradual expansion of the outer raceway, which is the end of the heat preload loop for spindle bearings. In the remainder of the study, the transient contact analysis will be referred to as bearing contact analysis (Section 2.2.).

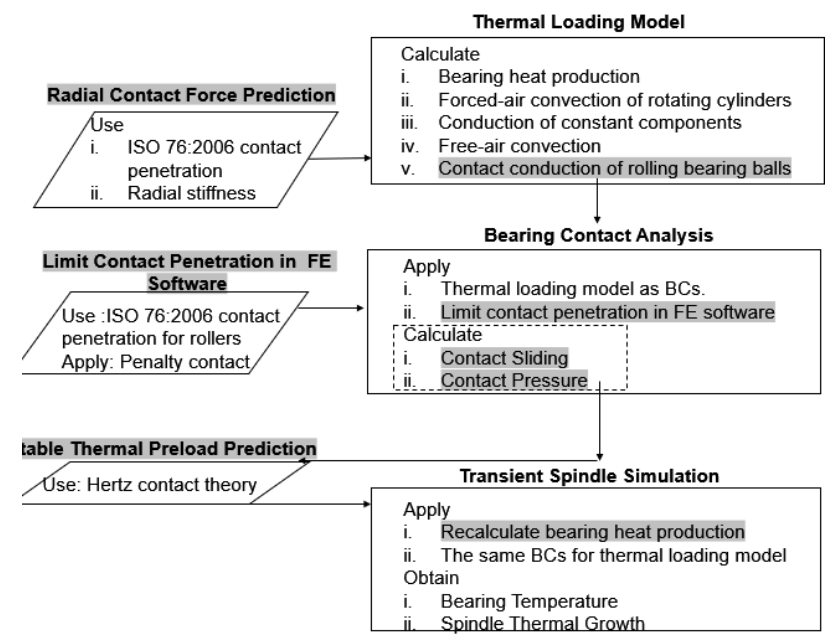

Fig. 1: Flowchart of the proposed method

The selection of the proper boundary conditions is significant for bearing contact analysis to predict precise thermal deflection and preload variation of bearings. The boundary conditions are imposed onto bearing assemblies in an equivalent form of the case in a spindle structure without a shaft or bearing cooling system. The boundary conditions include the following: i) Bearing heat generation due to friction, ii) Forced-air convection of rotating cylinders (spindle shaft, etc.), iii) Conduction of stationary components, iv) Free-air convection, v) Contact conduction of rolling bearing elements. The details of the boundary conditions will be given in Section 2.1 and be referred to as the thermal loading model. The bearing contact analysis leads to the prediction of steady preload variation of bearings. Subsequently, the predicted preload variation is employed on a spindle assembly to detect the spindle bearing temperature and thermal growth. The details of the transient spindle simulation are shared in Section 2.3. Fig.1 shows the proposed FE framework in this study. As seen from Fig.1, the contact penetration under the radial contact force is used to determine the contact conductivity of rolling bearing balls in the thermal loading model. This information is employed to calculate the contact sliding and pressure in 
bearing contact analysis. Moreover, predicted contact penetration (according to ISO-76:2006, it should be 0.01\% of the rolling element diameter) is used to limit the maximum nodal penetration amount between the contacting pairs in the FE model to obtain a precise contact sliding and pressure. Subsequently, the FE contact data are processed via Hertz contact theory to predict steady thermal preload for bearings. This prediction is used in the recalculation of bearing heat generation for transient spindle simulation.

\subsection{Thermal Loading Model}

Thermal loading model includes the proper selection of boundary conditions for bearing contact analyses and transient spindle simulation. The built-in motor heat generation is only considered in transient spindle simulation. Thus, the motor heat effects are excluded from this section, and it is assumed that there is no cooling system for internal spindle shaft or bearings. As mentioned, bearing assemblies (bearings and spacers) are modeled for bearing contact analyses, and the selected boundary conditions are imposed. According to these assumptions, the boundary conditions are as follows.

i) Bearing heat generation due to friction: Harris' model [13] for bearing heat generation is employed in this study. This model includes the heat generation due to friction torque and viscous friction. The details of the model are given in the Appendix. Friction torque calculation requires the bearing preload which is the most influential variable in a bearing assembly. Thus, the heat partitioning in a bearing assembly is based on the preload distribution according to the bearing configuration. This boundary condition is imposed on bearing raceways.

ii) Forced-air convection of rotating cylinders: Forced-air convection increases the heat transfer between the ambient air and the rotating cylindrical components, i.e., shaft, drawbar, etc. For this, first, the Reynolds number for the surrounding air of a rotating cylinder is calculated [13]. The calculation details are also given in the Appendix. The forced-air convection coefficient depends on the spindle speed and the rotating shaft diameter. Since the spindle shaft diameter is in general variable along its axis, the forced-air convection coefficient is calculated locally.

iii) Conduction of stationary components: In this method, the steel conduction condition is applied to the inner and outer raceways of the bearings. The conduction coefficient of each component is assigned according to the material properties in the transient spindle analysis.

iv) Free-air convection: Free-air convection is taken from the FE library.

v) Contact conduction of rolling elements: Since contact surfaces are in a rotating frame, and experience quasistatic contact, the contact conduction of rolling elements is a significant element for bearing contact analysis, and thus simulation of transient spindle behavior. A rise in contact pressure increases the contact conduction since the contact area enlarges. As mentioned earlier, contact penetration creates a radial contact force acting on bearing rolling elements which depends on diameter of rolling elements according to ISO-76:2006. This radial force is independent of the other variables and leads to higher contact conduction coefficients as it increases the contact pressure. Furthermore, the rotating bearing frame creates centrifugal forces in the radial direction increasing the contact area, and thus the contact conduction coefficients further. The bearing heat generation or dissipation may become dominant depending on the contact situation. An increase in contact pressure increases the contact conduction causing higher heat dissipation between the contacting pairs. On the other hand, higher contact pressure and spindle speed also accelerates the heat generation. Therefore, the bearing heat generation or dissipation mechanism becomes more dominant according to variables that affect the bearing temperature and preload variation. The dominant heat mechanism sequence was described in the preload heat feedback loop given in Introduction. According to this sequence, the contact conduction coefficient of rotating bearing elements is obtained from experimental data generated under different radial contact forces and spindle speeds [8]. An extrapolated graph from [8] is illustrated in the Appendix., First, the radial contact force on a bearing ball $\left(F_{r}\right)$ should be calculated in order to determine the conduction coefficient from the data given in [8]:

$$
F_{r}=K_{r} p_{a}
$$

The radial rigidity of a bearing assembly $\left(K_{r}\right)$ is given as a standard information in bearing manufacturer catalogs for different bearing configurations [15]. The contact penetration $\left(p_{a}\right)$ is predefined for spindle bearing manufacturers according to ISO-76:2006 which is $0,01 \%$ of the rolling element diameters. Therefore, this physical contact penetration $\left(p_{a}\right)$ is employed to limit the maximum nodal penetration amount between the contacting pairs in a FE model of bearing assembly. The contact modeling in FE [6] is based on Hertz-Signorini-Moreau (HSM) conditions where the penetration amount between a bearing ball and raceway is constrained in a contact analysis.

\subsection{Bearing Contact Analysis}

Transient contact analyses in FE software require high computational effort as a typical spindle assembly consists of approximately 1500 contacting pairs. Therefore, only the bearing assembly (bearings and spacers) is modeled in FE to predict preload by a transient thermo-structural model. The thermal loading model (Section 2.1) is employed in the bearing contact analysis. The applied thermal loading causes bearing race expansion and distortions at the center of a bearing element seized between inner and outer races. These distortions result in changes in contact displacements (penetration and sliding) and contact pressure causing bearing preload variations. As mentioned, contact penetration is defined in [7], and this physical contact penetration is required to limit in FEA for contacting node pairs in the proposed method. Contact sliding is directly associated with friction and boots the preload heat feedback loop. Thus, contact displacements are used to predict thermally induced preload variations of ball bearings. According to the Hertz contact theory, the contact pressure profile exhibits a concave down parabolic variation for point and line contacts [14]. The benchmark contact stress tests in FE software [6] targets the same pressure profile as Hertz's contact. Therefore, the average of the pressure inside the bearing rolling elements is calculated for the bearing contact analysis to estimate an average thermally induced pressure changes in spindle bearings.

$$
\begin{aligned}
K_{\text {tpreload }} & =Q_{\text {avg }} / \delta^{3 / 2} \\
F_{\text {tpreload }} & =K_{\text {tpreload }} \delta_{\text {sliding }}
\end{aligned}
$$

Bearing contact analysis results are employed to obtain thermally induced preload variation in bearings via Hertz contact theory [14] by employing Eqns.2 and 3. Eqn.3 leads to bearing preload variation. In Eqn. 2, the average pressure of bearing balls $\left(Q_{\text {avg }}\right)$ is accepted as the radial thermally induced pressure in spindle bearings, and $\left(\delta_{\text {sliding }}\right)$ stands for contact sliding. From the Hertz contact theory, one can obtain thermally induced stiffness 
$\left(K_{\text {tpreload }}\right)$ and bearing preload variation $\left(F_{\text {tpreload }}\right)$ by employing Eqn.3. The bearing contact analysis involves a transient FEA where the bearing preload variation exhibits a nonlinear behavior until the outer bearing raceways start expanding. To reduce the computational effort, steady (linear) preload variation is considered, where the outer bearing raceways expand, for transient spindle analysis.

\subsection{Transient Spindle Simulation}

The thermal load model introduced in Section 2.1 is used to calculate the total heat to be applied on the entire spindle assembly. After the bearing contact analyses, steady bearing preload variation is predicted and used to recalculate bearing heat generation. Additionally, power losses from the built-in motor are imposed on transient thermal FEA. Subsequently, the thermal load history is imported to structural analysis for thermal growth calculations of the spindle.

\section{APPLICATION}

\subsection{FE models}

The proposed method was applied to a spindle designed and manufactured by Igrek Makina Inc. The spindle design aims for high torque and rigidity. The only cooling system in the spindle is for the refrigeration of the built-in motor. Configuration of the angular contact bearings is DBD [15] for the front and DB [15] for the front and rear spindle bearings, respectively. The bearing model is NSK 100 BNR10X (L) for the front bearings while NSK75BNR 75 $10 X(L)$ is employed for the rear bearings [15]. As the lubricant, grease is used. The material of bearing balls is ceramic where the raceways are steel. Fig.2 illustrates the spindle structure. FE analyses completed via ANSYS where the penalty method is employed for contacting bodies with the maximum allowed penetration of $0.01 \%$ of the bearing ball diameter. The spindle assembly was meshed with tetrahedral elements except for the bearing balls for which pyramid meshes were used. For analyses of the front bearing assembly, 118,093 nodes and 58,970 mesh elements were used in total whereas 43,106 nodes and 21,732 mesh elements were used for the rear bearing assembly. For transient spindle analyses, 1,0761,09 nodes and 591,507 mesh elements were used. The computation time was 45 minutes for the transient analyses. All boundary conditions were applied with constant spindle speed of $10,000 \mathrm{rpm}$.

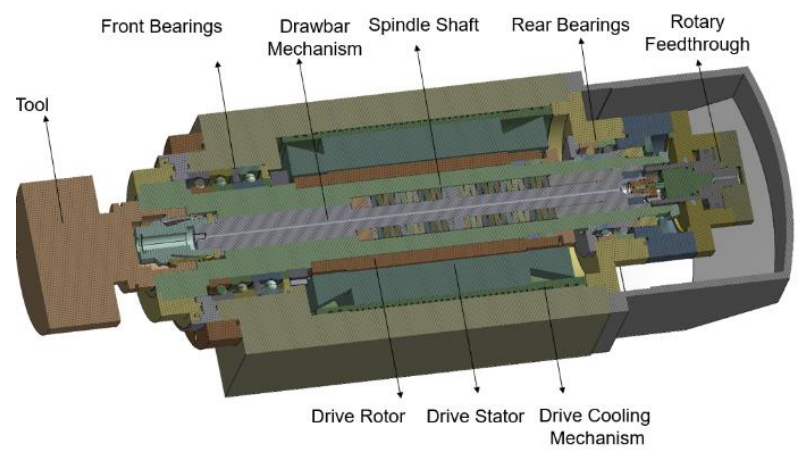

Fig. 2: The spindle structure.

\subsection{Thermal Loading Model}

First, the boundary conditions (thermal loading model) were determined for the spindle bearings. The initial step was the calculation of the bearing heat generation as indicated in Section 2.1. The calculated results presented in Fig.3.and Fig.3.b illustrate the total heat generated by the front and rear spindle bearings, respectively, up to $10,000 \mathrm{rpm}$. The heat partitioning between the raceways is calculated according to relative inner and outer raceway masses [16] The initial preloads applied were $529 \mathrm{~N}$ and $269 \mathrm{~N}$ for the front and rear bearing assemblies, respectively.

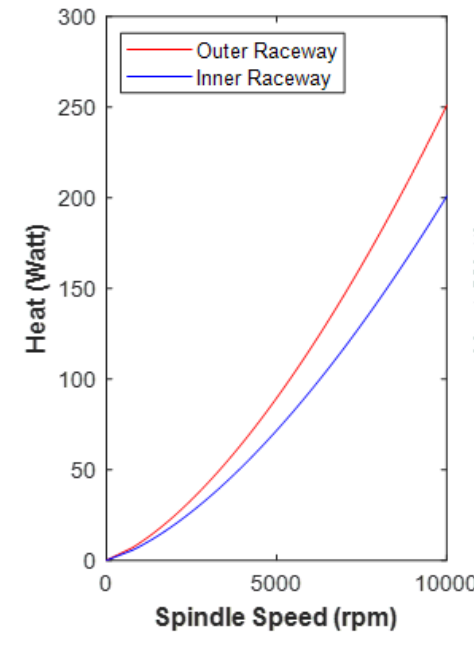

(a)

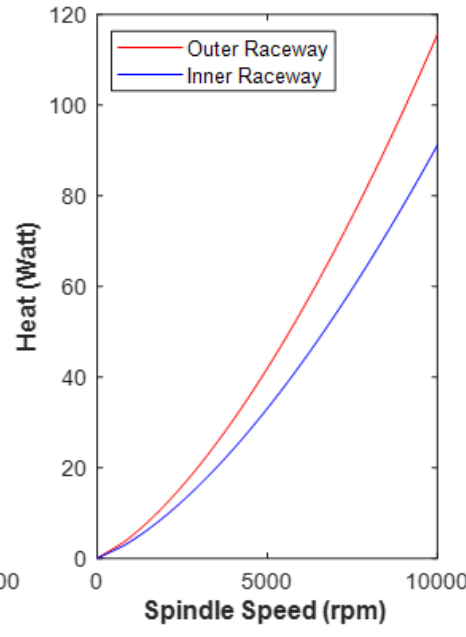

(b)
Fig. 3: Heat distribution of (a) front and (b) rear bearing assembly.

The rotating cylinder forced-air convection coefficient was calculated by following the procedure given in Section 2.1. In order to obtain the contact conduction coefficient [8], Fig. A1 from the Appendix was used. The front bearing radial force $\left(F_{r}\right)$ was obtained by using the radial rigidity of a bearing assembly $\left(K_{r}\right)$ in the bearing catalogs [15] and was factorized for DBD configuration of the front bearings. Similarly, the rear bearing radial force $\left(F_{r}\right)$ was found by using the radial rigidity of a bearing assembly $\left(K_{r}\right)$ in the bearing catalogs and was factorized for DB configuration for rear bearings.

The steel conduction coefficient is applied to the inner and outer raceways of ball bearings. The boundary conditions used are shown in Table 1 for the bearings. The preload distribution is unequal for DBD bearing configuration. The preload of first bearing is higher than the other bearings.

Tab. 1: Front and rear bearings assembly boundary conditions.

\begin{tabular}{ccccc}
\hline Bearings & $\begin{array}{c}\boldsymbol{F}_{\boldsymbol{r}} \\
(\mathbf{N})\end{array}$ & $\begin{array}{c}\text { Contact } \\
\text { Cond. Of } \\
\text { Bearings } \\
\left(\mathbf{W} / \mathbf{m}^{2} \mathbf{~ K}\right)\end{array}$ & $\begin{array}{c}\text { Forced-air } \\
\text { Conv. of } \\
\text { Shafts } \\
\left(\mathbf{W} / \mathbf{m}^{2} \mathbf{K}\right)\end{array}$ & $\begin{array}{c}\text { Const. } \\
\text { Element } \\
\text { Cond. } \\
(\mathbf{W} / \mathbf{m ~ K})\end{array}$ \\
\hline Front \#1 & 20 & 520 & 99 & \\
Front \#2\&3 & 10 & 470 & 99 & 54.7 \\
Rear \#1\&2 & 9.5 & 465 & 86 & \\
\hline
\end{tabular}

\subsection{Bearing Contact Analysis}

In this section, the bearing heat calculated from Harris'model (with catalog preload) which is one of the boundary conditions presented in Section 3.2 for the FEA. This heat applied to raceways of the bearings for a 45minute transient bearing contact analyses. Thermal expansion of bearings occurs due to heat production and changes the location of the contact point. Because of this change, the preload changes and need to be recalculated. 
This value is recalculated (based on Eqns.2 and 3 (Hertz contact theory)) by using the average contact stress and displacement that are obtained from the FE simulation. It is worth to mention that the contact displacement value is taken from contact sliding card in ANSYS contact tool. Later, this recalculated (thermally induced) preload is used again in Harris' heat generation model to simulate the entire spindle structure in Section 3.4.

The point contacts on ball surfaces should be checked during the FE analyses as a reliability test of the contact analyses which is may generally cause convergence problems [6]. The thermal contact analysis results of bearings are shown in Fig. 4.

a.) Pressure profile on the coincident b.) Pressure profile on the coincident surface with outer raceway surface with inner raceway
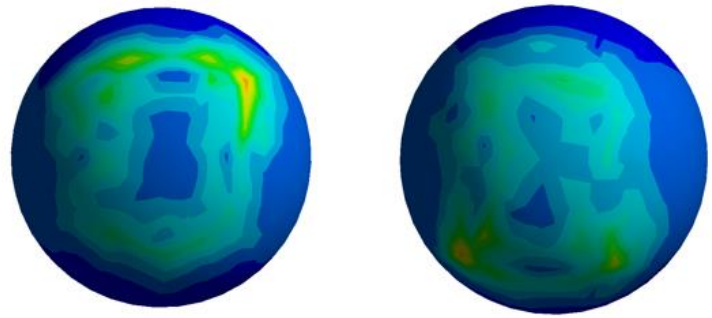

Fig. 4: Point contact pressure on bearing ball surfaces.

Thermally induced contact pressure analysis results are illustrated in Fig.5 for the front bearings. The maximum contact pressure on the bearing ball surface is $17.8 \mathrm{MPa}$ as can be seen in Fig.5. The average contact pressure due to thermal loading is $8.94 \mathrm{MPa}$ for the front bearings. Fig.6 illustrates that the contact sliding is $0.408 \mu \mathrm{m}$ for the front bearings. Fig.7 illustrates the results of thermally induced contact pressure analysis for the rear bearings. The maximum contact pressure on the bearing ball surface is 5.42 MPa as shown in Fig.7. The average contact pressure due to thermal loading is $3.62 \mathrm{MPa}$ on the rear bearings. Fig. 8 illustrates that the contact sliding is $0.168 \mu \mathrm{m}$ for the rear bearings.
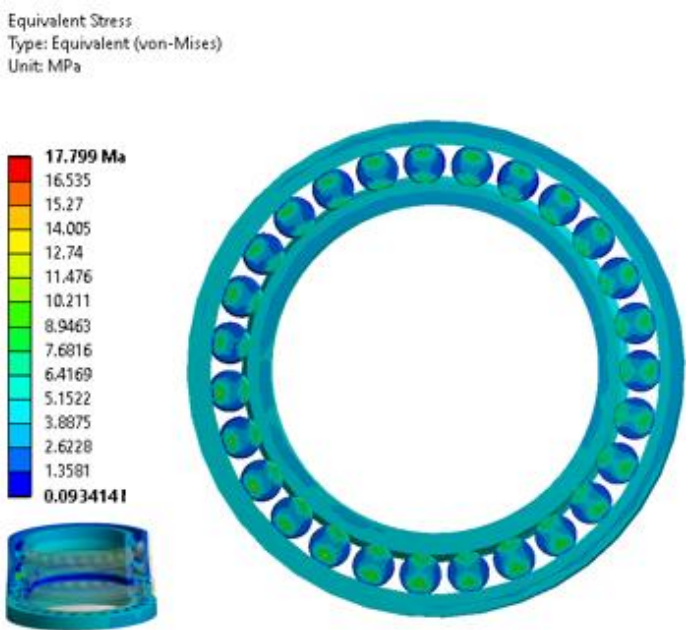

Fig. 5: Thermally induced contact pressure of front bearings.
CONTSUD Expression: CONTSUID Unit: $\mu \mathrm{m}$

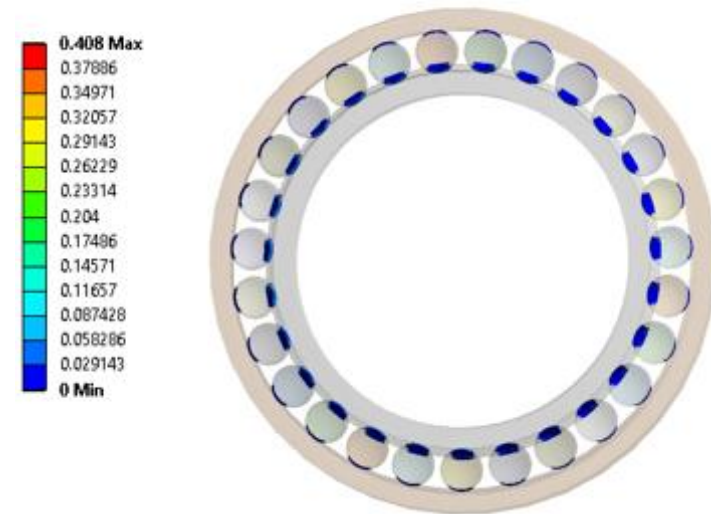

Fig. 6: Thermally induced contact sliding of front bearings.

The thermally induced preload was calculated as $1396 \mathrm{~N}$ for the front bearings while the initial preload was $389 \mathrm{~N}$ for DB/DF configuration and $529 \mathrm{~N}$ for DBD configuration. Similarly, the thermal preload was calculated as $905 \mathrm{~N}$ for the rear bearings while the initial preload was 289 for DB/DF configuration.

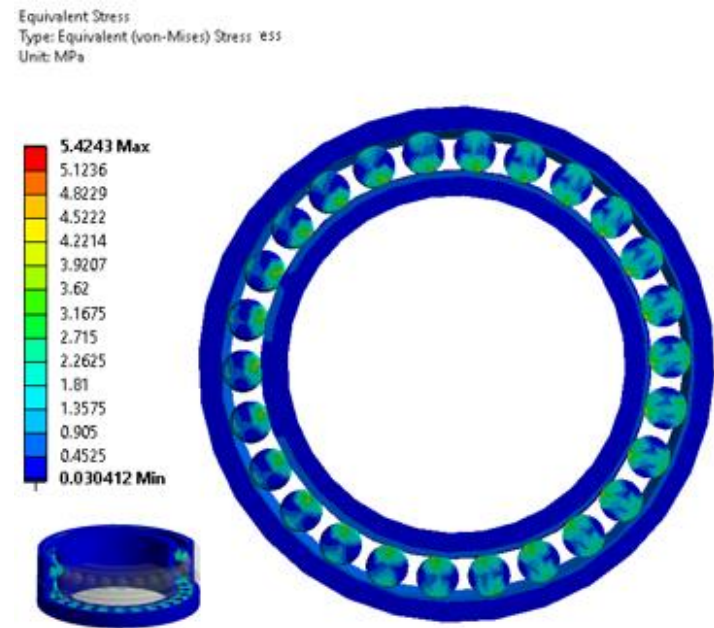

Fig. 7: Thermally induced contact pressure of rear bearings.

CONTSUID
Expression: CONTSLID
Uniti um

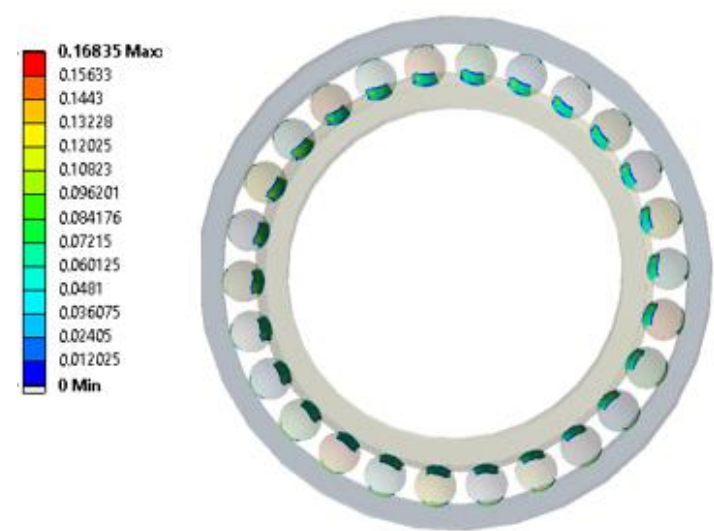

Fig. 8: Thermally induced contact sliding of rear bearings. 


\subsection{Transient Spindle Simulation}

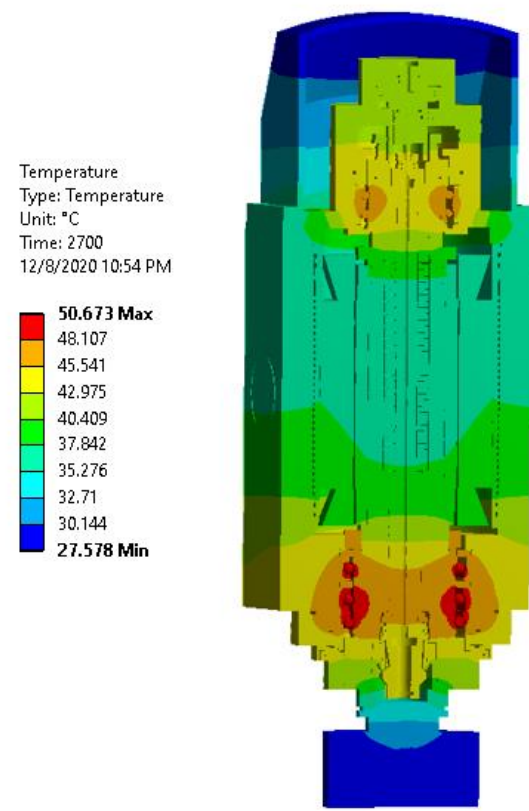

Fig. 9: The temperature distribution obtained from the FE analysis.

The transient spindle simulation is a coupled thermomechanical analysis. First, thermal FE model was constructed to obtain the temperature results for the spindle assembly. Then, this temperature results were imported to the structural FE analysis to obtain thermal growth for the entire assembly. The entire spindle assembly was loaded thermally by applying the calculated steady thermal preload and other boundary conditions in the transient thermal FEA. In FEA, steel conductivity $(54.7 \mathrm{~W} / \mathrm{m} \mathrm{K})$ was assigned to the spindle housing and its' surrounding structure as conduction boundary conditions. Convection boundary conditions were imposed on the outer surface of the spindle housing by using the stagnant airflow available in the ANSYS library. Subsequently, the obtained thermal loads were imported to structural analysis for predicting thermal growth of the spindle. The results are shown in Fig.9 for transient thermal analysis. The structural analysis results indicate the maximum thermal growth was $87.8 \mu \mathrm{m}$ for Zaxis, while $36.8 \mu \mathrm{m}$ for $X$-axis and $42.4 \mu \mathrm{m}$ for $Y$-axis on the spindle.

\section{NUMERICAL RESULTS AND EXPERIMENTAL VERIFICATION}

\subsection{Experiments}

Fig.10 illustrates the experimental setup for measurement of the thermal growth during the tests. Bearing temperatures were measured by thermocouples installed on the outer raceways of ball bearings. Spindle thermal growth (extension) was measured by capacitive displacement sensors as shown in Fig. 10.

The total test period was 60 minutes. In the first 15 minutes, the spindle speed was increased from 0 to $10,000 \mathrm{rpm}$ gradually. The initial bearing temperature was $18^{\circ} \mathrm{C}$ at stationary condition. At the end of the 15-minute speeding up period, the bearing temperature rose to $28^{\circ} \mathrm{C}$. According to the bearing manufacturer catalogs [15], a $10^{\circ} \mathrm{C}$ change is an expected result when the speed increases from 0 to
$10,000 \mathrm{rpm}$. Bearing temperature and spindle growth were measured when spindle was running at $10,000 \mathrm{rpm}$. For these measurements, the test period was 45 minutes in total.

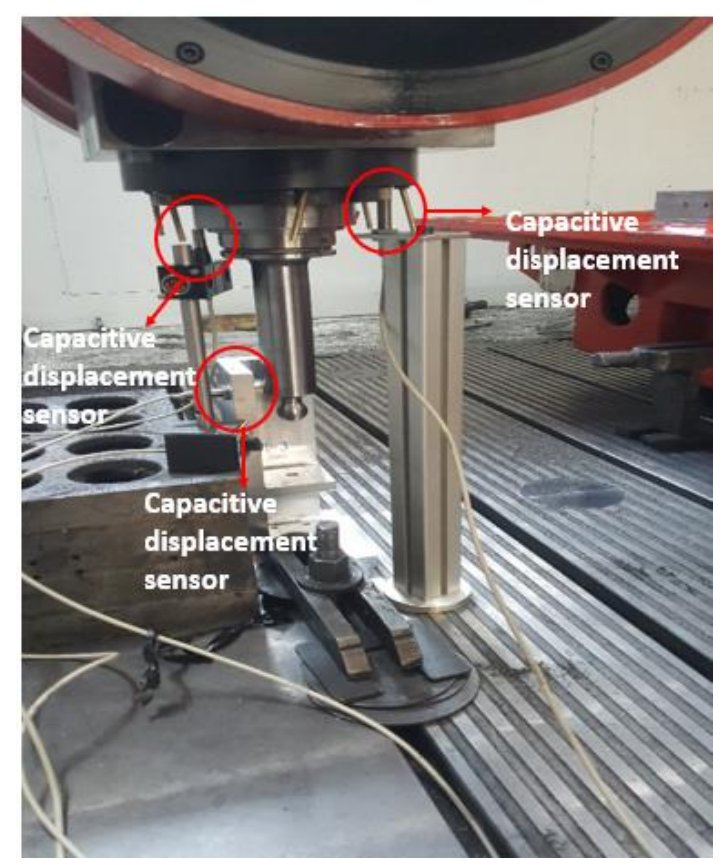

Fig. 10: The experimental setup.

\subsection{Bearing Temperature Results}

The comparison of the FE analysis and experimental results is given in Fig.11. According to Fig.11, the discrepancy is relatively high for the first 20 minutes since the steady thermal preload was employed in the simulations. The total analysis solution time was 24 hours for the FE model on a computer with 128GB ram and Intel Xeon Gold $6136 \mathrm{CPU}$ (with 2 processors in total.). The discrepancy may be reduced by applying the transient history of the thermal bearing preloads. However, this transient history application would increase the analysis solution time significantly due to required iterations until convergence. On the other hand, a reasonable agreement can be seen after the first 30 minutes of the operation. Therefore, the proposed modeling approach provides acceptable trends for the steady state spindle bearing temperatures.

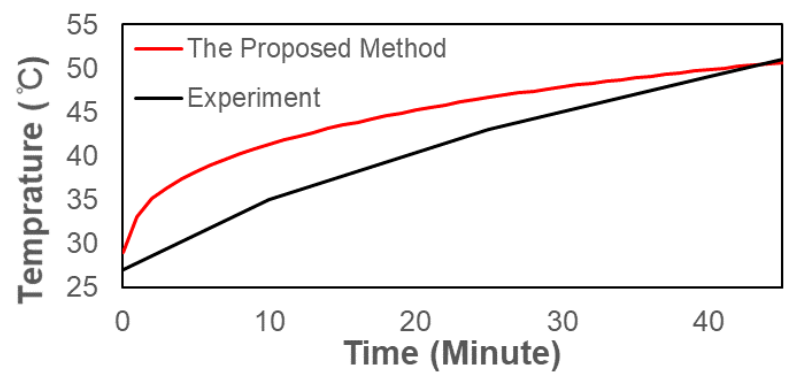

Fig. 11: The comparison of experimental and FE results for bearing temperature.

\subsection{Spindle Thermal Growth Results}

The FE results for the spindle growth are shared in Fig.12. The FE probe results (local area results) were adjusted according to the measurement points of the capacitive displacement sensors. Fig.12 indicates the thermally induced deformations in all 3 axes after 45 mins of operation. 


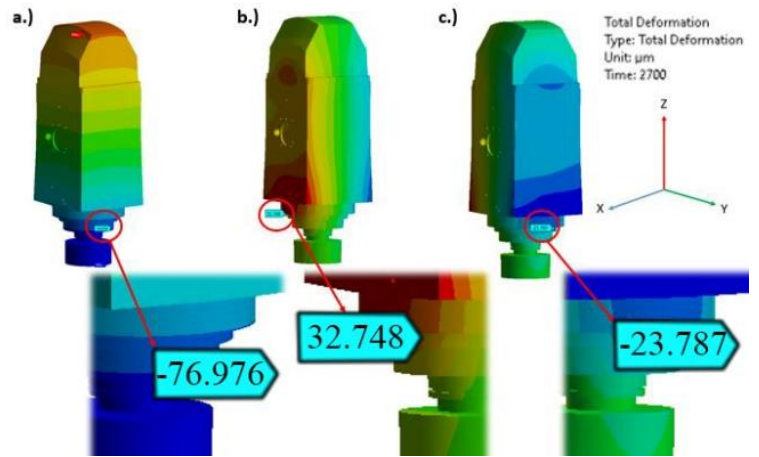

Fig. 12: The FE results for thermal growth in (a) Z-axis, (b) $X$-axis, (c) $Y$-axis after 45 mins of operation.

The Z-axis thermal growth was investigated by employing the prosed methods in FE and conducting experiments on the manufactured prototype. The experimental and simulation results are illustrated in Fig.13. indicating around $13 \%$ difference after the first 30 minutes.

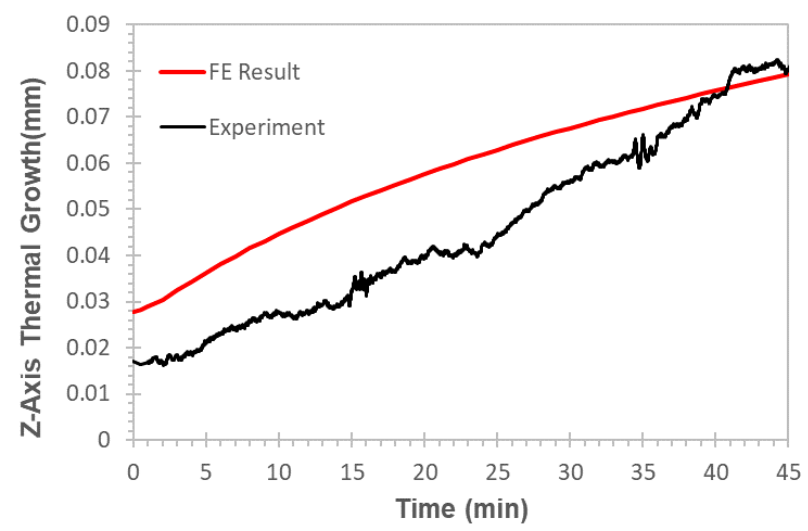

Fig. 13: The experimental and simulation results for thermal growth in Z-axis.

Similarly, the $\mathrm{X}$-axis thermal displacement was investigated by comparing the proposed method predictions and the measurements. Fig.14 illustrates the results for the $\mathrm{X}$-axis where the maximum difference of about $4 \%$ with the FE results is observed after the first 30 minutes.

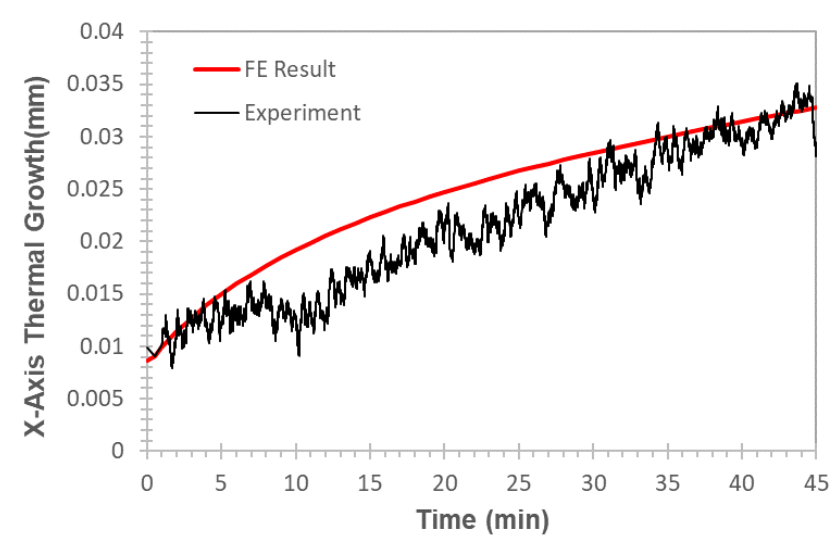

Fig. 14: The experimental and simulation results for thermal displacements in $X$-axis.

The $Y$-axis thermal displacements are given in Fig.15. The maximum discrepancy with the predictions is around \%14. All thermal deformation predictions for the first 30 minutes of the operation obtained by the proposed method have less than $20 \%$ discrepancy with the measurements which is considered as a reasonable approximation for the early spindle design stage. The discrepancies reduce down to $10 \%$ when the steady state is reached.

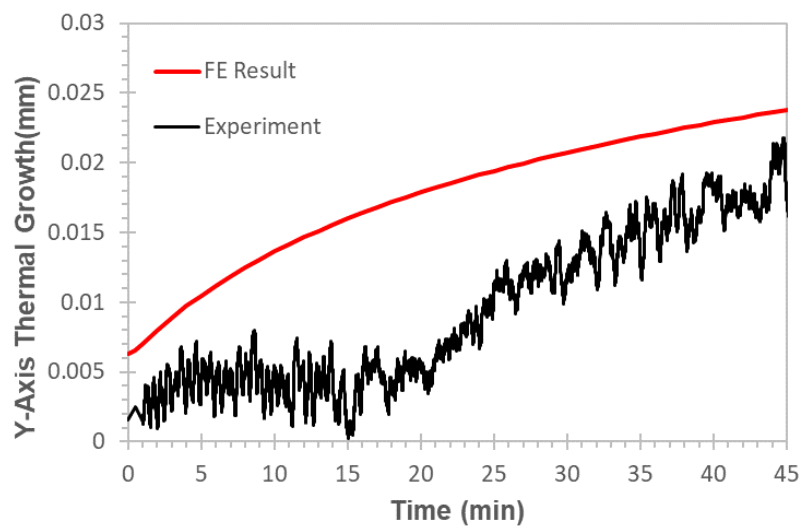

Fig. 15: The experimental and simulation results for thermal displacements in $Y$-axis.

These results are expected, i.e., the discrepancy with the measurements reduce as the time progresses, since the steady-state conditions of the thermally induced preload is taken for heat generation calculations for transient spindle analyses as well.

\section{CONCLUSION}

In this study, an FE framework is proposed to predict bearing temperature and spindle thermal growth on spindle bearings. The method includes a thermal loading model and a transient contact analysis. In the contact analysis, bearing contact deformations (penetration and sliding) and pressure are predicted by considering contact algorithms in an FE software. A transient spindle simulation in FE is employed to predict the bearing temperature and thermal spindle growth. The method results compared with the experimental ones. The bearing temperature measurements and thermal deformation tests were conducted on the manufactured spindle prototype. The results indicate a reasonable performance. The method does not require any experiments and provides reasonable accuracy for predictions after 40 minutes, since the steadystate values of the thermally induced preload is used for the heat calculation in the FE simulations. Therefore, it could be a useful tool for thermo-structural spindle analyses at the early design stage. From these analyses, bearing configuration and lubricant selection can be improved. Furthermore, cooling system can also be optimized based on the predictions from the proposed method. Therefore, the proposed method and the procedure can be used to improve different parts of a spindle during the design stage.

\section{ACKNOWLEDGMENTS}

This research was supported by the Scientific and Technological Research Council of Turkey (TÜBITAK) under the TEYDEB project number 1190124.

\section{REFERENCES}

[1] Mayr, J., Jedrzejewski, J., Uhlmann, E., Donmez, M. A., Knapp, W., Härtig, F., ... \& Brecher, C. (2012). Thermal issues in machine tools. CIRP annals, 61(2), 771-791.

[2] Li, Hongqi, and Yung C. Shin. "Analysis of bearing configuration effects on high-speed spindles using an integrated dynamic thermo-mechanical spindle model." International Journal of Machine Tools and Manufacture 44.4 (2004): 347-364. 
[3] Abele, Eberhard, Yusuf Altintas, and Christian Brecher. "Machine tool spindle units." CIRP annals 59.2 (2010): 781 802.

[4] Shillor, Meir, Mircea Sofonea, and Józef Joachim Telega. Models and analysis of quasistatic contact: variational methods. Vol. 655. Springer Science \& Business Media, 2004.

[5] Klarbring, A. "Examples of non-uniqueness and nonexistence of solutions to quasistatic contact problems with friction." Ingenieur-Archiv 60.8 (1990): 529-541.

[6] Wriggers, P., \& Zavarise, G. (2004). Computational contact mechanics. Encyclopedia of computational mechanics.

[7] ISO 76: 2006. "Rolling bearings—static load ratings." (2006).

[8] Liu, J., Ma, C., \& Wang, S. (2020). Thermal contact conductance between rollers and bearing rings. International Journal of Thermal Sciences, 147, 106140.

[9] Yan, K., Yan, B., Wang, Y., Hong, J., \& Zhang, J. (2018). Study on thermal induced preload of ball bearing with temperature compensation based on state observer approach. The International Journal of Advanced Manufacturing Technology, 94(9-12), 3029-3040.

[10] Zhang, K., Wang, Z., Bai, X., Shi, H., \& Wang, Q. (2020). Effect of preload on the dynamic characteristics of ceramic bearings based on a dynamic thermal coupling model. Advances in Mechanical Engineering, 12(1), 1687814020903851.

[11] Li, Hongqi, and Yung C. Shin. "Integrated dynamic thermo-mechanical modeling of high-speed spindles, part 1: model development." J. Manuf. Sci. Eng. 126.1 (2004): 148-158.

[12] Holkup, T., Cao, H., Kolář, P., Altintas, Y., \& Zelený, J. (2010). Thermo-mechanical model of spindles. CIRP annals, 59(1), 365-368.

[13] Harris, T. A. (2001). Rolling bearing analysis. John Wiley and sons.

[14] Johnson, K. L., \& Johnson, K. L. (1987). Contact mechanics. Cambridge university press.

[15] Catalogue, N. B. (2011) Super Precision Bearings NSK.

[16] Ashby, M. F., Abulawi, J., \& Kong, H. S. (1991). Temperature maps for frictional heating in dry sliding. Tribology Transactions, 34(4), 577-587.

\section{APPENDIX}

\subsection{Harris' Model for Bearing Heat Generation}

According to the bearing heat model, one needs to calculate friction torque $\left(M_{\text {load }}\right)$ due to bearing loads. $F_{\beta}$ stands for an equivalent dynamic load that composed of axial $\left(F_{a}\right)$ and radial $\left(F_{r}\right)$ force in Eqn. 1. $F_{\beta}$ yields $F_{a}$ for vertical spindles. $d_{m}$ is the average bearing diameter.

$$
M_{\text {load }}=F_{\beta} f_{1} d_{m}
$$

Axial force $\left(F_{a}\right)$ is affected by the gravitational load $\left(F_{\text {grav }}\right)$ and bearing preload $\left(F_{\text {preload }}\right)$ for angular ball bearings.

$$
F_{a}=F_{\text {grav }}+F_{\text {preload }}
$$

$f 1$ is the bearing coefficient based on static equivalent load $\left(P_{o}\right)$ and basic static load rating $\left(C_{0}\right)$ in which $z$ and $y$ values depend on the bearing type [13].

$$
\mathrm{f}_{1}=z\left(P_{o} / C_{0}\right)^{y}
$$

Overall bearing heat $\left(H_{\text {load }}\right)$ is calculated by the following empirical formula [13]

$$
H_{\text {load }}=\left(1.047 \times 10^{-4}\right) n M_{\text {load }}
$$

Lubricant viscous behavior is another frictional contributor One needs to calculate viscous friction torque ( $\left.M_{\text {viscous }}\right)$ using the kinematic viscosity of the bearing lubricant $\left(v_{o}\right)$ and bearing coefficient $\left(f_{0}\right)$ [13].

$$
\begin{array}{llll}
M_{\text {viscous }}=f_{o}\left(v_{o} n\right)^{2 / 3} d_{m}{ }^{3} 10^{-7} & \text { if } & v_{o} n \geq 2000 \\
M_{\text {viscous }}=160 \times 10^{-7} f_{o} d_{m}{ }^{3} & \text { if } & v_{o} n \leq 2000
\end{array}
$$

Inertial loading occurs between the rolling elements and the bearing raceways due to the orbital and rotational speeds at high speeds. Therefore, for better prediction accuracy one needs to add inertia effects to Eqn.A5.[13] which are neglected in this study due to the moderate spindle speed under investigation.

Overall bearing heat for viscous behavior $\left(H_{\text {viscous }}\right)$ can be calculated similar to $H_{\text {load }}$ in eqn. A4.

$$
H_{\text {viscous }}=\left(1.047 \times 10^{-4}\right) n M_{\text {viscous }}
$$

Then, the total heat generated in the spindle bearings is the summation of both contributors:

$$
H_{\text {total }}=H_{\text {load }}+H_{\text {viscous }}
$$

\subsection{Forced-air Convection of Rotating Cylinders}

Reynolds number $(R e)$ can be calculated as

$$
R e=u_{\text {fluid }} l_{\text {shaft }} / v_{\text {fluid }}
$$

where $u_{\text {fluid }}$ and $v_{\text {fluid }}$ are the velocity and the kinematic viscosity of the ambient air flowing through the spindle unit, respectively. In calculation of convection coefficients for a cylindrical surface, the perimeter of the cylinder ( $l_{\text {shaft }}$ and the velocity of the ambient air $u$ fluid are used:

$$
\begin{aligned}
& l_{\text {shaft }}=\pi d_{\text {shaft }} \\
& u_{\text {fluid }}=l_{\text {shaft }} n / 60
\end{aligned}
$$

The Prandtl number $(\mathrm{Pr})$ which is a material property for the air and calculated as 0.707 at $25^{\circ} \mathrm{C}$ in the previous studies [13] whereas Nusselt number $(N u)$ is defined as

$$
N u=0.0225 \operatorname{Re}^{4 / 5} \operatorname{Pr}^{0.3}
$$

The convective heat transfer coefficient, $h_{\text {shaft }}$ can be calculated by considering conditions in different spindle speeds as follows:

$$
h_{\text {shaft }}=N u v_{\text {fluid }} / l_{\text {shaft }}
$$

\subsection{Contact Conduction of Rolling Bearing Balls}

Fig. A1 extracted from the experimental data given in Ref.8 for a spindle ball bearing under different radial forces.

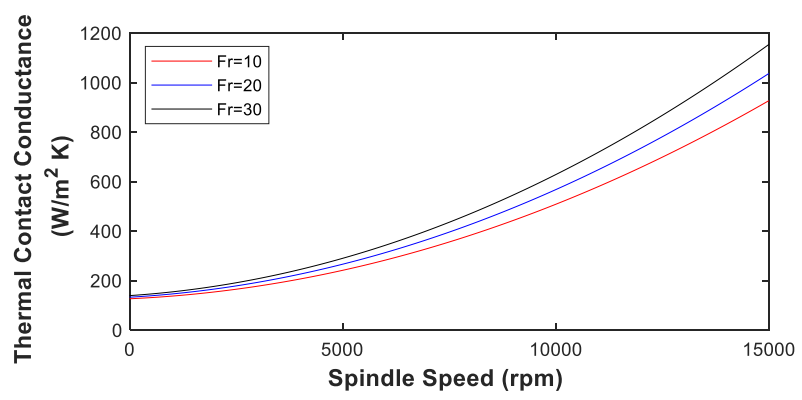

Fig. A1: Contact Conductance and Spindle Speed Relation Under a Radial Force on a Spindle Bearing [8]. 